\title{
Splice variants of zinc finger protein 695 mRNA associated to ovarian cancer
}

\author{
Sergio Juárez-Méndez ${ }^{1}$, Alejandro Zentella-Dehesa ${ }^{3,4}$, Vanessa Villegas-Ruíz ${ }^{5}$, Oscar Alberto Pérez-González ${ }^{1}$ \\ Mauricio Salcedo ${ }^{5}$, Ricardo López-Romero ${ }^{5}$, Edgar Román-Basaure ${ }^{7}$, Minerva Lazos-Ochoa ${ }^{6}$, \\ Víctor Edén Montes de Oca-Fuentes ${ }^{4}$, Guelaguetza Vázquez-Ortiz ${ }^{5,8^{*}+}$ and José Moreno ${ }^{2^{*} \dagger}$
}

\begin{abstract}
Background: Studies of alternative mRNA splicing (AS) in health and disease have yet to yield the complete picture of protein diversity and its role in physiology and pathology. Some forms of cancer appear to be associated to certain alternative mRNA splice variants, but their role in the cancer development and outcome is unclear.

Methods: We examined AS profiles by means of whole genome exon expression microarrays (Affymetrix GeneChip 1.0) in ovarian tumors and ovarian cancer-derived cell lines, compared to healthy ovarian tissue. Alternatively spliced genes expressed predominantly in ovarian tumors and cell lines were confirmed by RT-PCR.

Results: Among several significantly overexpressed AS genes in malignant ovarian tumors and ovarian cancer cell lines, the most significant one was that of the zinc finger protein ZNF695, with two previously unknown mRNA splice variants identified in ovarian tumors and cell lines. The identity of ZNF695 AS variants was confirmed by cloning and sequencing of the amplicons obtained from ovarian cancer tissue and cell lines.

Conclusions: Alternative ZNF695 mRNA splicing could be a marker of ovarian cancer with possible implications on its pathogenesis.
\end{abstract}

Keywords: Ovarian cancer, Alternative mRNA splicing, ZNF695

\section{Background}

Ovarian Cancer (OC) is the sixth most prevalent form of cancer worldwide, which has a high mortality rate because at the time of diagnosis nearly $70 \%$ of cases are at an advanced stage, leading to a 5 year survival below 30\% [1]. The classification of OC depends on its cellular origin, with approximately two-thirds belonging to the epithelial serous type [2]. Similar to other types of cancer, OC is characterized by changes in gene expression profiles [3-6], including under and overexpression, or even de novo gene expression $[7,8]$.

Alternative splicing (AS) provides a critical and flexible layer of regulation, intervening in many biological processes, such as the diversity of proteins.

\footnotetext{
* Correspondence: vazquezortizg@niiddk.nih.gov; jmoreno49@gmail.com ${ }^{\dagger}$ Equal contributors

${ }^{5}$ Genomic Oncology Laboratory, Medical Research Unit in Oncologic Diseases, Oncology Hospital, National Medical Center Siglo XXI, IMSS, Mexico City, Mexico

${ }^{2}$ Mexican Faculty of Medicine, Universidad La Salle, Mexico City, Mexico Full list of author information is available at the end of the article
}

AS has major impact on the cell phenotype as a single pre-mRNA spliced in different ways can give rise to different mature mRNA transcripts (variants) that are translated onto distinct proteins varying in functions [9-11]. Apparently, over $90 \%$ of human genes have two or more splice variants $[9,10]$, greatly increasing the complexity of both the transcriptome and the proteome [12,13]. Therefore, AS could play an important role in gene regulation both in health and disease. In cancer, AS could affect the cellular processes related to tumor progression, including inhibition of apoptosis, tumor invasiveness, metastasis and angiogenesis [14].

Among the genes with well established AS patterns whose derived alternative proteins affect tumor cell behavior is the SRPK1 kinase that in breast, colonic and pancreatic carcinomas phosphorylates the splicing factor SF2/ASF, allowing import to the nucleus, where it modulates AS of multiple target mRNAs, such as BIN1, S6K1, MNK2, contributing to tumor progression [15,16]. Additional cancer types with apparent alterations of alternative

\section{Biomed Central}

(c) 2013 Juárez-Méndez et al.; licensee BioMed Central Ltd. This is an Open Access article distributed under the terms of the Creative Commons Attribution License (http://creativecommons.org/licenses/by/2.0), which permits unrestricted use, distribution, and reproduction in any medium, provided the original work is properly cited. 
splicing, include: gastric, colon and bladder carcinomas [17], hepatocarcinoma [18], prostatic cancer [19,20], multiple myeloma [21], breast cancer, and OC [22], where most cancer-associated transcript variants belong to genes related to processes such as cellular transformation [23,24], adhesion, proliferation, migration and invasion [25-28]. In OC, a new, previously unknown, variant of p53 mRNA transcript variant (p538) was identified [29] whereas variants of the NR4A1, a nuclear receptor involved in steroidogenesis, and MRRF, a mitochondrial protein, were identified in prostatic cancer [20]. Although the extent and pathophysiological meaning of this has yet to be established, there is little doubt that the study of alternative splicing can lead to a better understanding of the mechanisms of cancer development, and to the identification of new biomarkers for the diagnosis, epidemiological studies of prevalence, prognosis, and therapeutic responses.

The aim of the present study was to identify the whole genome profile of alternatively spliced mRNA in ovarian cancer and cell lines by high-density microarrays. Among the spectrum of several ovarian cancer-associated alternatively spliced genes, one mRNA, coding for ZNF695, a zinc finger protein, had the most significantly overexpression in OC with two prominent splice variants that were not present in normal ovarian tissue. These variants were cloned and sequenced. Here we describe some of the characteristics of ZNF695 mRNA splicing variants associated to ovarian cancer.

\section{Methods}

\section{Data set and specimens}

All investigations were performed in accordance with the Declaration of Helsinki with approval by the Central Research Committee of the Mexican Institute of Social Security and The Ethics Committee of Centro Médico Siglo XXI, Mexican Institute of Social Security. After informed consent was obtained, normal ovarian tissue (HOT), borderline ovarian tumor (BOT), malignant epithelial ovarian tumors stages III and IV (MOT) tissues were collected by the clinical partners at the Oncology Hospital, National Medical Center Siglo XXI, IMSS, and at the General Hospital of Mexico SSA (Secretaría de Salud) from patients with diagnosed ovarian cancer, or healthy ovarian tissue from patients who underwent abdominal surgery for hysterectomy due to uterine myomatosis with no evidence of ovarian pathology. Routinely, during this type of procedure, in patients over 45 years old both ovaries are removed, and only one in patients under 45.

Cancer and corresponding normal tissue specimens were cut into three fragments and snapped frozen in liquid nitrogen, one of which was stored in RNA Latter ${ }^{\mathrm{R}}$ (Qiagen, Valencia, CA, USA) at $-70^{\circ} \mathrm{C}$ for a maximum of two months until RNA was purified, and the other two remaining fragments were formalin-fixed, paraffin-embedded, sliced, mounted on slides, and stained with HE. Only tissue samples with $>80 \%$ tumor cells or normal epithelial cells (MOT or HOT, respectively), according to the histopathological examination were included for analysis.

Moreover, we also included for study ovarian cancer cell lines (OCL) NIH: OVCAR-3 [30], SK-OV-3 [31], TOV112D [32] and TOV-21G [32], kindly provided by Dr. Laura Díaz-Cueto, Research Unit on Reproductive Medicine, Instituto Mexicano del Seguro Social (IMSS).

Samples were disrupted using a TissueLyser ${ }^{\mathrm{Tw}}$ system (Qiagen, Valencia, CA, USA) for 60s at $30 \mathrm{~Hz}$. Total RNA was obtained with RNeasy Mini Kit (Qiagen, Valencia, CA, USA) and total RNA concentration was quantified using a NanoDrop ND-1000 spectrophotometer and RNA quality was visualized and measured on an Agilent RNA 6000 Nano Assays in an Agilent 2100 Bioanalyzer (Agilent Technologies, Santa Clara, CA, USA).

\section{Microarray GeneChip 1.0 assay}

The microarray used for these studies was Affymetrix GeneChip 1.0, which contains over 750,000 probe sets representing all exons of $\sim 28,800$ annotated genes. Sample amplification and preparation for microarray hybridization was performed according to Affymetrix specifications (http://media.affymetrix.com/support/downloads/manuals/ wt_expressionkit_manual.pdf). In brief, $100 \mathrm{ng}$ total RNA was reverse transcribed to cDNA, amplified by in vitro transcription and reverse transcribed to cDNA again. Fragments between 40 and 70 bp were generated enzymatically, labelled and hybridized onto the microarray chips in an Affymetrix hybridization oven at $60 \mathrm{rpms}$, $45^{\circ} \mathrm{C}$ for 17 hours. Chips were washed according to the established protocols (Affymetrix, Santa Clara, CA, USA) with GeneChip fluidics station 450, and finally they were scanned with an Affymetrix 7G GeneChip scanner. The raw data (CEL files) will be deposited in Gene Expression Omnibus (GEO).

\section{Data analysis}

Microarray analysis was achieved by means of CEL files of the Partek Genomics Suite $6.5 \mathrm{v}$ software (Partek Incorporated, Saint Louis, MO). Probe sets were summarized by means of Median Polish and normalized by quantiles with no probe sets excluded from analysis. Background noise correction was achieved by means Robust Multi-chip Average (RMA) and data were $\log 2$ transformed. Data grouping and categorization was achieved by principal component analysis (PCA). Differentially expressed exons were detected by means of Alternative Splicing ANOVA with the healthy control samples as the baseline. Moreover, BOT, MOT and OCL were also examined against HOT by the Geometric least squares means model. Hierarchical clustering was based on the 
dissimilarity of samples (Euclidian method) by means of average linkage.

\section{Reverse transcription PCR}

For linear cDNA synthesis, $1 \mu \mathrm{g}$ total RNA was predigested with 1 U DNAse, $1 \times$ DNAse buffer, $5 \mathrm{mM}$ EDTA, after which it was incubated at $37^{\circ} \mathrm{C}$ for $30 \mathrm{~min}$ and at $65^{\circ} \mathrm{C}$ for an additional $10 \mathrm{~min}$. Thereafter, samples were placed in master mix containing: $40 \mathrm{U}$ Ribolock RNAse inhibitor, $0.2 \mu \mathrm{g}$ random hexamer primers, $20 \mathrm{mM}$ dNTP's mix, $40 \mathrm{U}$ M-Mulv reverse transcriptase (RT), and $1 \times$ M-Mulv RT Buffer (Thermo Scientific).

Conditions for endpoint PCR amplification were: 5' CGAATGAgAgCTGGCAAAGGCAAA 3' Fwd., 5' ACGCCAAGTGCCGTACAATTCATC 3' Rev. primers (housekeeping gene RPL4) $7.5 \mathrm{mM}, 1 \times$ Taq buffer, $2 \mathrm{mM}$ $\mathrm{MgCl}_{2}, 0.4 \mathrm{mM}$ dNTP's, $1.25 \mathrm{U}$ Taq Pol, 2 ul cDNA; whereas ZNF695 was amplified with primers: 5' GCCT TTGTCTCCTTGCGGC 3' Fwd. 5' GGCTGTCTTCTCT GTGTTCACGTT 3' Rev. 12.5 mM, $1 \times$ Taq buffer, $3 \mathrm{mM}$ $\mathrm{MgCl}_{2}, 0.4 \mathrm{mM}$ dNTP's, $1.25 \mathrm{U}$ Taq Pol, 2 ul cDNA. In both cases mix reactions were initially incubated at $95^{\circ} \mathrm{C}$ for $5 \mathrm{~min}$, and then were run for 40 cycles at $94^{\circ} \mathrm{C} 45 \mathrm{~s}$, $59^{\circ} \mathrm{C} 45 \mathrm{~s}, 72^{\circ} \mathrm{C} 60 \mathrm{~s}$; and finally at $72^{\circ} \mathrm{C}$ for $5 \mathrm{~min}$.

\section{PCR product purification and cloning}

PCR products were separated by electrophoresis (2.5\% agarose gels) and extracted by means of Gel extraction kit ${ }^{\mathrm{TM}}$ (Qiagen, Valencia, CA, USA). The extracted products were ligated into pGem-T Easy Vector ${ }^{\mathrm{rm}}$ (Promega, Madinson, WI) by incubating overnight in $1.5 \mathrm{~mL}$ Eppendorf tubes with $2 \times$ Rapid Ligation Buffer (T4 ligase), pGEM-T Easy Vector, PCR product and T4 DNA ligase at $4^{\circ} \mathrm{C}$.

Recombinant plasmid DNA was purified with Wizard Plus Miniprep DNA Purification System ${ }^{\text {Tx }}$ (Promega) and selected clones were sequenced with M13 oligonucleotide and BigDye Terminator 3.1 cycle sequencing kit (Applied Biosystems), and sequenced in an Applied Biosystems Abi Prism 3130 genetic analyzer automated sequencer. Subsequently, the PCR amplicon sequences were assembled and checked against the transcript sequences annotated in the NCBI nucleotide database.

\section{Results}

\section{Expression microarray assays}

A total of 14 samples with an RNA integrity number $(\mathrm{RIN}) \geq 8$ were hybridized in GeneChip 1.0 microarrays according to the MIAME guidelines. Histopathological classification of tissues was as follows: healthy ovarian tissue $(\mathrm{HOT}) \mathrm{n}=4$, benign ovarian tumors $(\mathrm{BOT}) \mathrm{n}=2$, (malignant) serous epithelial ovarian tumors in stages III and IV (MOT) $\mathrm{n}=4$, and ovarian cell lines (OCL) $\mathrm{n}=4$. As a prerequisite, healthy tissue had to be free of any visible alteration, whereas all tumor tissues, benign or malignant, selected for study contained at least $90 \%$ tumor cells.

Background correction and normalization of microarrays reported no quality control errors (QC) and, as expected, the $\mathrm{QC}$ intergroup proportions were variable (Additional file 1), whereas gene expression histograms were similar in all samples. General gene expression was examined and visualized according to the histological groups by means of PCA. As expected, except for HOT and BOT that essentially overlapped, MOT and OCL clustered in distinct regions of the PCA plot (Figure 1). Thus, HOT and BOT clustered together in the negative end, whereas MOT and OCL clustered separately in the positive area. This indicates that our data set has the power to discriminate $\mathrm{OC}$ (both MOT and OCL) from normal tissue or benign tumors. Moreover, as expected, OC gene expression profiles show a wide dispersion, reflecting tumor heterogeneity. In contrast, HOT and BOT plotted in relatively close proximity, even at the level of individual samples that for the two BOT samples practically overlapped. Finally, as expected, HOT also had some individual sample variability, probably reflecting proportional differences of tissue contents, individual variability or variations in the estrous stage, neither of which was addressed.

Moreover, hierarchical clustering on the basis of relative gene expression also grouped HOT and BOT together, whereas malignancies (both MOT and OCL) clustered together but distant of HOT and BOT (Figure 2). Nonetheless, careful analysis of individual genes revealed some small intragroup differences that could reflect tumor heterogeneity and that deserves further in depth analysis. Interestingly, predominant $\mathrm{OC}$-associated changes in gene expression were suppression rather than overexpression (Additional file 2).

Up to here, the results show two major ovarian tissue gene expression patterns, one specific of $\mathrm{OC}$ (cell lines and tumors) and the other one characteristic of borderline tumors and healthy tissue. The gross differences seen probably reflect the relatedness among the different groups. To further explore this, we compared gene expression by grouping apparently related conditions together, against each of the individual or grouped opposites (Table 1). By these means, the highest differences were MOT + OCL vs. BOT + HOT $(\mathrm{n}=1799)$, followed by MOT + OCL vs. HOT (1498 differences), MOT + OCL vs. BOT (1030 differences), BOT + MOT + OCL vs. HOT (545 differences). Finally, gene expression differences between BOT and HOT were minimal ( 28). On the basis of these findings, we chose to further examine the most significant differentially expressed exons.

\section{Exon analysis identifies two major ovarian cancer-associated,} differentially spliced transcripts of gene ZNF695

Once we had examined the relative OC-associated gene expression profiles, it was important to examine whether 


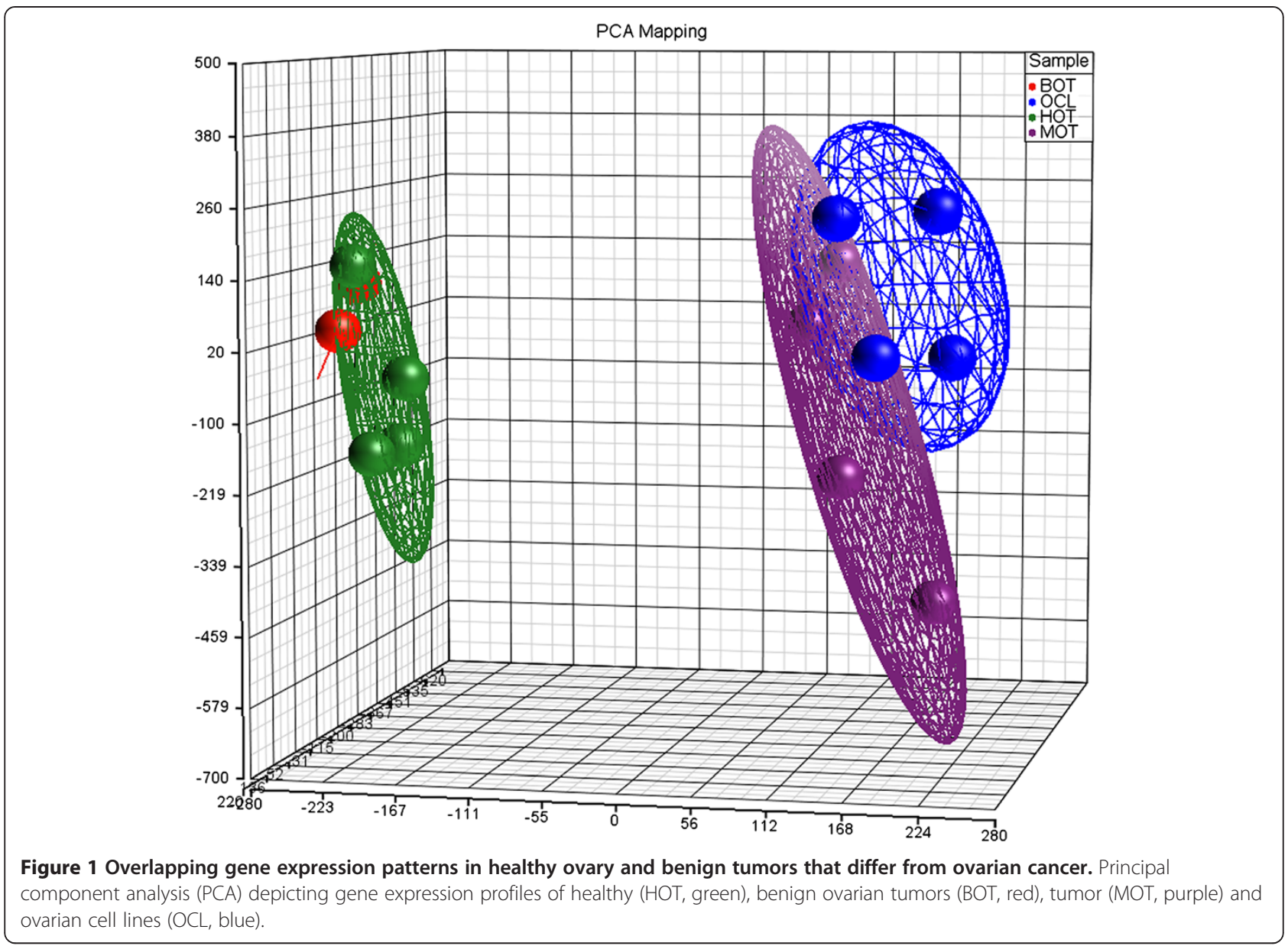

some of the overexpressed genes reflected only quantitative differences or if there were also qualitative differences among them. To achieve this, we performed exon analysis of genes overexpressed in both MOT and OCL. As differential exon usage cannot be easily examined in suppressed genes, we exclusively examined overexpressed genes.

The analysis was performed by means of Alternative Splicing ANOVA and the criteria to select genes for exon analysis were: false discovery ratio (FDR) $<0.05$ and fold change $>3$, in at least one probe set. According to these criteria, the number of overexpressed alternatively spliced genes in OC was 207 (Additional file 3). To identify OCpredominant splice variants, these genes were subjected to internal analysis by comparing the expression of each individual exon for each study group against the mean total expression of the same gene in each of the groups, and were considered only when they yielded $\geq 3$ fold change. Moreover, we performed visual inspection of each of the individual exon expression profile graphs $(\mathrm{MOT}+\mathrm{OCL}$ vs. HOT + BOT or vs. HOT or vs. BOT, all of which yielded the same genes). This procedure identified at least nine genes of potential interest (Table 2), of which, ZNF695 (encoding a zinc-finger protein) had the highest overexpression $(\sim 7$ fold, FDR $<0.005)$ in MOT and OCL, and its mRNA had the highest significant changes in exon expression with significant suppression in one its exons when compared to HOT and BOT (Figure 3). The remaining of this study focuses on the characterization of ZNF695.

\section{ZNF695 splice variants in OC}

ZNF695 encode a zinc finger protein with as yet unknown functions and its gene contains six exons located in chromosome 1q cytogenetic positions 247,148,625$247,171,358$. This gene has six possible transcripts of which two (ZNF695-003, and ZNF695-006) encode complete ORFs yielding a 515 and a 172 amino acid length proteins, respectively; whereas the other four transcripts encode products thought to undergo nonsensemediated decay, a process that detects nonsense mutations and prevents the expression of truncated transcripts (http://www.ensembl.org/Human/Search/Results?q=ZNF6 95;site=ensembl;facet_species=Human).

To characterize transcripts expressed predominantly in $\mathrm{OC}$, we designed primers to identify and clone splice variants of ZNF695 most likely corresponding to the message lengths preferentially expressed in the four initial OC 

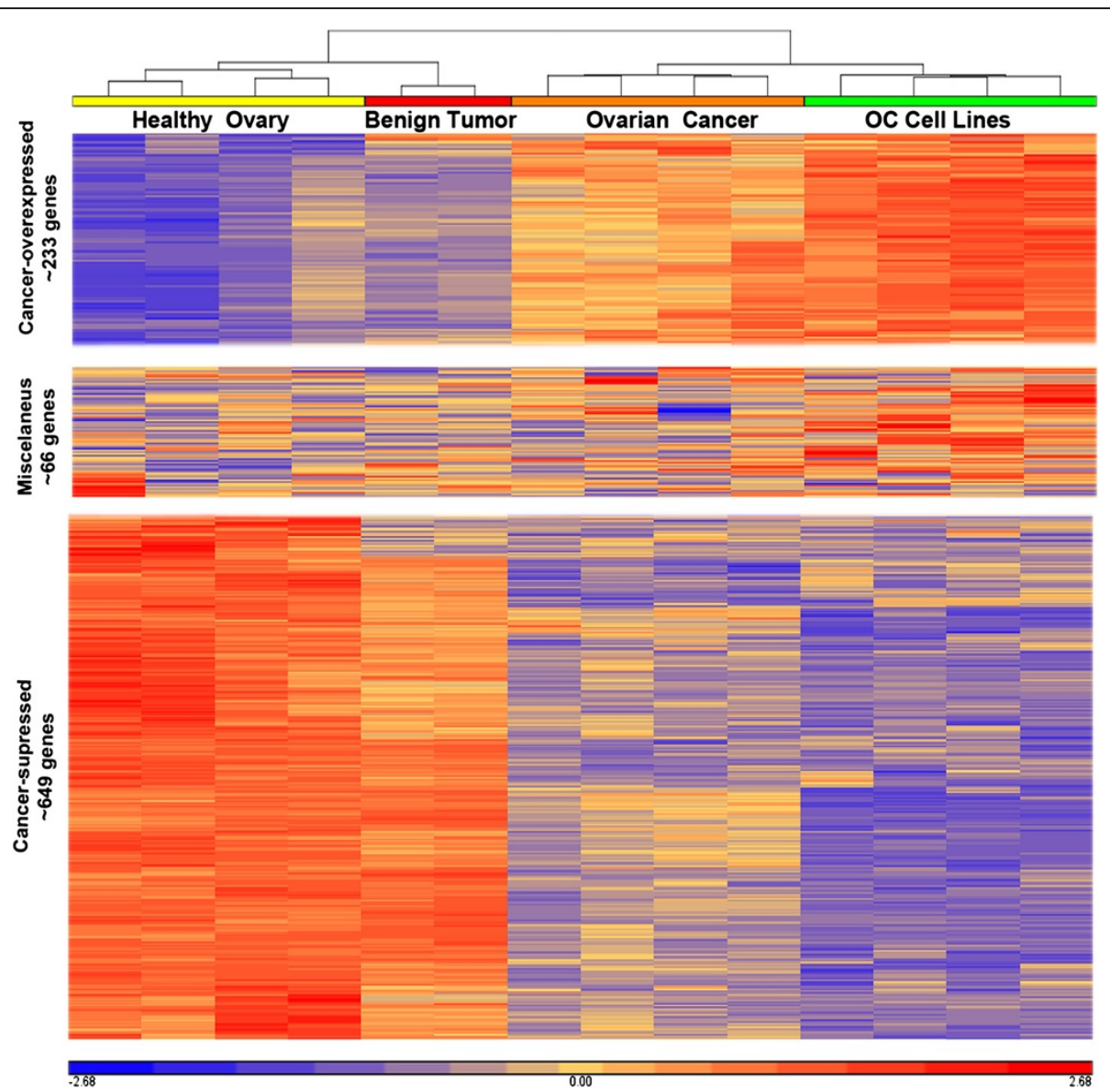

Figure 2 Hierarchical clustering of ovarian cancer gene. Heat maps depicting hierarchical clustering of gene expression in healthy ovarian tissue, benign ovarian tumours, ovarian cancer and ovarian cancer cell lines. Graphics were generated by means of Partek Genomics Suite ${ }^{\mathrm{TM}}$ software. Differentially expressed gene values were included only when fold-change was $>3$ and false discovery ratio $<0.005$ and only the number of genes clustering to over- expression (top), no significant changes (middle) and under-expression (bottom) are shown. Gene names, RefSeq, and other details are shown in Additional file 2.

Table 1 Gene expression differences among groups

\begin{tabular}{cccc}
\hline Comparisons & $\begin{array}{c}\text { No. } \\
\text { differences }\end{array}$ & Over-expressed & Suppressed \\
\hline BOT vs. HOT & 28 & 24 & 4 \\
MOT vs. HOT & 1329 & 476 & 853 \\
OCL vs. HOT & 1664 & 669 & 995 \\
MOT vs. BOT & 625 & 135 & 490 \\
OCL vs. BOT & 1369 & 488 & 881 \\
OCL vs. MOT & 666 & 385 & 281 \\
BOT + MOT + OCL vs. HOT & 545 & 146 & 399 \\
MOT + OCL vs. HOT & 1498 & 508 & 990 \\
MOT + OCL vs. BOT & 1030 & 262 & 768 \\
HOT + MOT + OCL vs. BOT & 368 & 28 & 340 \\
MOT + OCL vs. BOT + HOT & 1799 & 595 & 1205 \\
\hline
\end{tabular}

Table 2 Main genes whit potential alternative splicing

\begin{tabular}{ccccc}
\hline $\begin{array}{c}\text { \# of } \\
\text { markers }\end{array}$ & $\begin{array}{c}\text { Gene } \\
\text { symbol }\end{array}$ & $\begin{array}{c}\text { p-value } \\
\text { (sample) }\end{array}$ & $\begin{array}{c}\text { Alt-splicing } \\
\text { (sample) }\end{array}$ & $\begin{array}{c}\text { Fold-Change(OCL and } \\
\text { MOT vs. BOT and HOT) }\end{array}$ \\
\hline 9 & ZNF695 & $1.28 E-05$ & $8.16 \mathrm{E}-05$ & 4.5411 \\
13 & CCNB1 & $3.11 \mathrm{E}-06$ & $2.26 \mathrm{E}-10$ & 4.98751 \\
36 & FANCD2 & $4.87 \mathrm{E}-05$ & $8.33 \mathrm{E}-10$ & 6.58464 \\
12 & CDKN3 & $2.51 \mathrm{E}-04$ & $7.59 \mathrm{E}-10$ & 5.42259 \\
25 & TPX2 & $1.05 \mathrm{E}-04$ & $2.04 \mathrm{E}-16$ & 11.0438 \\
14 & E2F8 & $1.27 \mathrm{E}-03$ & $1.92 \mathrm{E}-07$ & 3.20517 \\
14 & FOXM1 & $1.09 \mathrm{E}-04$ & $2.98 \mathrm{E}-08$ & 5.86201 \\
18 & HMMR & $1.71 \mathrm{E}-03$ & $3.87 \mathrm{E}-02$ & 6.55569 \\
50 & CENPE & $1.87 \mathrm{E}-03$ & $5.00 \mathrm{E}-05$ & 4.35735 \\
\hline
\end{tabular}



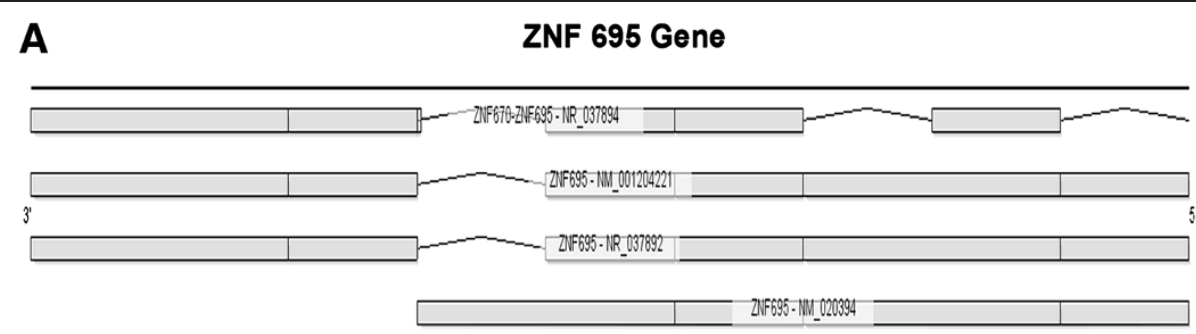

B

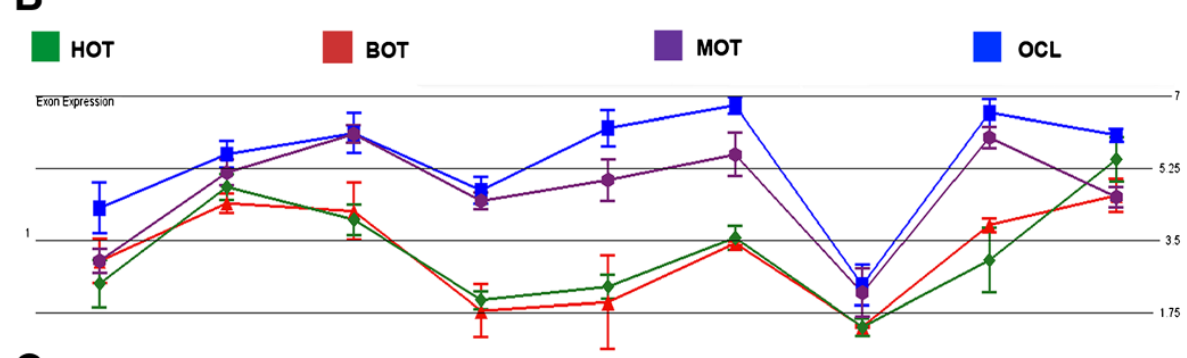

C

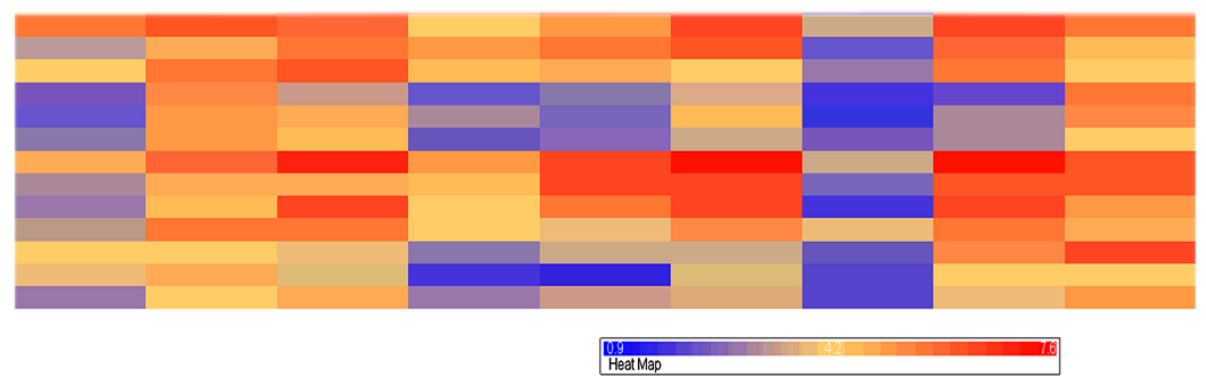

Figure 3 Differential ZNF695 exon usage in ovarian cancer. A) All possible splice variants of zinc finger protein (ZNF) 655 mRNAs retrieved from UCSC Genome Browser. B) Graphic depicting levels of individual exon expression in healthy ovarian tissue (HOT, green), benign ovarian tissue (BOT, red), malignant ovarian tumours (MOT, purple) and ovarian cancer cell lines (OCL, blue). C) Heat map of each of the peaks containing the individual samples.

samples. By means of RT-PCR, in a total of 14 OC tissues (10 MOT and four OCL), expression of the three different transcripts was as follows: seven out of 10 tumor samples and three out of four cell lines expressed all three transcripts at variable degree, whereas the remaining samples (three tumors and one cell line) only expressed the two larger transcripts (Figure 4A and B). All three amplicons were gel purified, cloned into pGEM-T Easy Vector (Figure 5A) and confirmed to correspond to the aforementioned ZNF695 RNA transcripts by sequencing (Figure 5B-E). The first one appears to correspond to the full-length product (FLP), as it yields a $400 \mathrm{bp}$ amplicon spanning FLP nucleotides 38 to 433 with full identity to ZNF695-003, ZNF695-006 and no other possible match (Figure 5B-C, Additional file 4). The second transcript spans 360 bp (that we name here ZNF695 transcript 4, see below) fully matching ENSEMBL nonsense-mediated decay transcript ZNF695-002, as well as NCBI peptide BAG54313.1 (Isogai, T. Helix Research Institute, Genomics Laboratory; e-mail: flj-cdna@nifty.com, http://www.ncbi.nlm. nih.gov/protein/193785160?from $=1 \&$ to $=118) \quad($ Figure 5B, E,
Additional file 5). The third transcript (named here ZNF695 transcript 5) found here aligns with both those transcripts up to nucleotide 361 , but our primers cannot identify further on the 5' direction. Although we cannot tell to which of ZNF695 splice variants it corresponds, this transcript contains a sequence partly identical to ZFN695-002 (ZNF695 transcript 4), except that it is missing a $52 \mathrm{bp}$ fragment containing part of the 5' untranslated region and misses the translation initiation signal (Figure 5D, E, Additional file 4). Although there are additional AUG codons as potential alternative translation initiation signals 3' of the canonical AUG, these fail to yield useful ORFs. Therefore, most likely, this transcript represents a long non-coding mRNA. Finally, Figure 5F shows the ZNF695 AS model according to the transcripts found in MOT and OCL.

\section{Discussion}

Understanding the origin of malignancy is one of the greater challenges of modern science. Among malignant tumors, $\mathrm{OC}$ represents a major problem because little is 
A RPL4 HOT MOT $(-)$ $300 \mathrm{bp}$ $200 \mathrm{bp}$

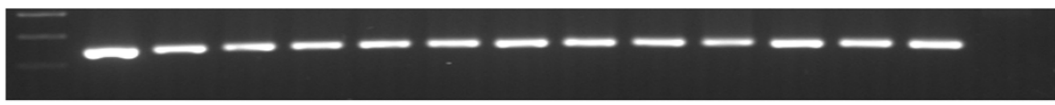
ZNF 695 $400 \mathrm{bp}$ 300 bp

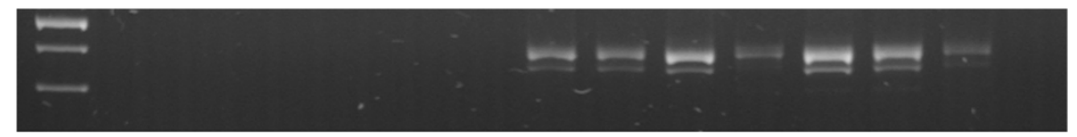

B

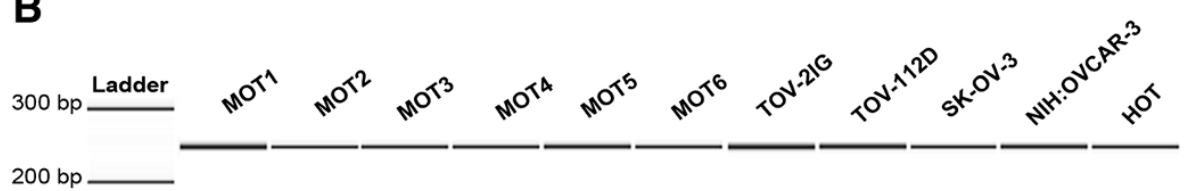
$200 \mathrm{bp}-\square$
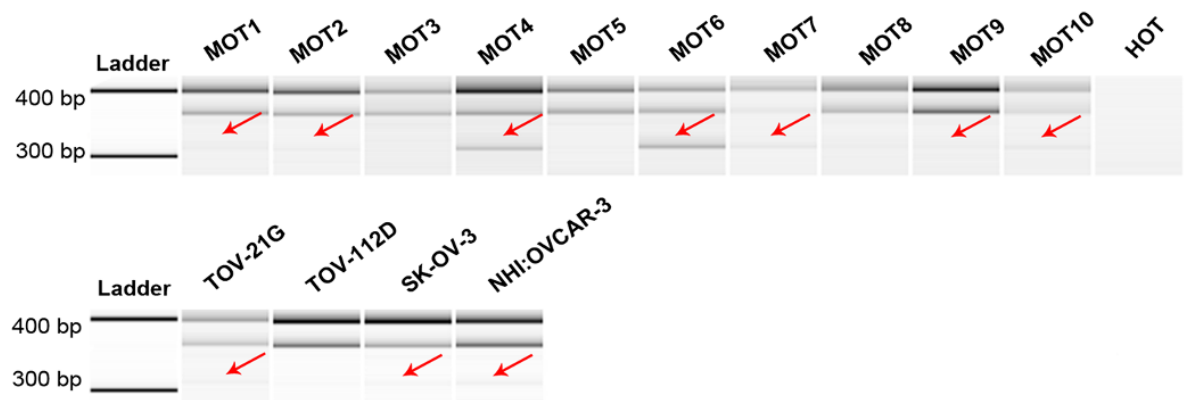

Figure 4 ZNF695 transcripts of healthy ovarian tissue, individual ovarian cancer samples, and cell lines. A) Agarose gel showing the raw RT-PCR products obtained from one HOT $(n=6)$ sample and MOT $(n=7)$ samples. Above, RPL4 protein (housekeeping), below, ZNF695. B) RT-PCR products run on a DNA Chip Agilent bioanalyzer. Top row: housekeeping gene RPL4. Middle and bottom rows: individual transcripts of each of the differentially spliced mRNAs in malignant ovarian cancer tissue $(M O T, n=10)$ and individual ovarian cancer cell lines ( $n=4$, shown by their names).

known about its pathogenesis, which is also difficult to identify in early stages as it goes asymptomatic over long periods of time to be detectable only in late stages, almost always beyond any possibility of remission [33,34]. Alternative exon splicing is a biological process of major importance, because gene changes leading to altered splicing can affect normal cell and tissue function $[19,20,27,35]$, including malignant transformation [36]. The current studies were carried out to examine whether OC could be associated to particular exon-splicing state and if so, to identify differentially spliced transcripts present in OC but absent in healthy ovarian tissue. With the exon array data set presented here, we identified nine overexpressed genes with differential exon profiles associated to OC, one such gene, ZNF695, coding for a largely uncharacterized zinc finger protein, is the most representative, with three transcripts differentially expressed by MOT and OCL, one corresponding to the whole protein, a second ORF corresponding to a shorter peptide and a third, with lower but significant expression that corresponds to a long non-coding mRNA. These results likely provide a useful biomarker of malignant transformation in women suspected to have OC and open the study of the role of these transcripts in cell proliferation and malignant transformation.

Alternative splicing is a major source of protein diversity, bioinformatics-based methods indicate that $>90 \%$ human genes could be subject to AS $[10,12,13]$ with an estimate of several million different proteins, and some individual proteins having over 1000 variants due only to AS [37]. This process can differ during distinct cellular functional or developmental stages $[38,39]$. It is, therefore, not surprising that AS has also been found altered during malignant transformation [40], which could be either a general marker of cancer or limited to certain cancer types. Moreover, cancer-associated AS could be the clue to understand the 


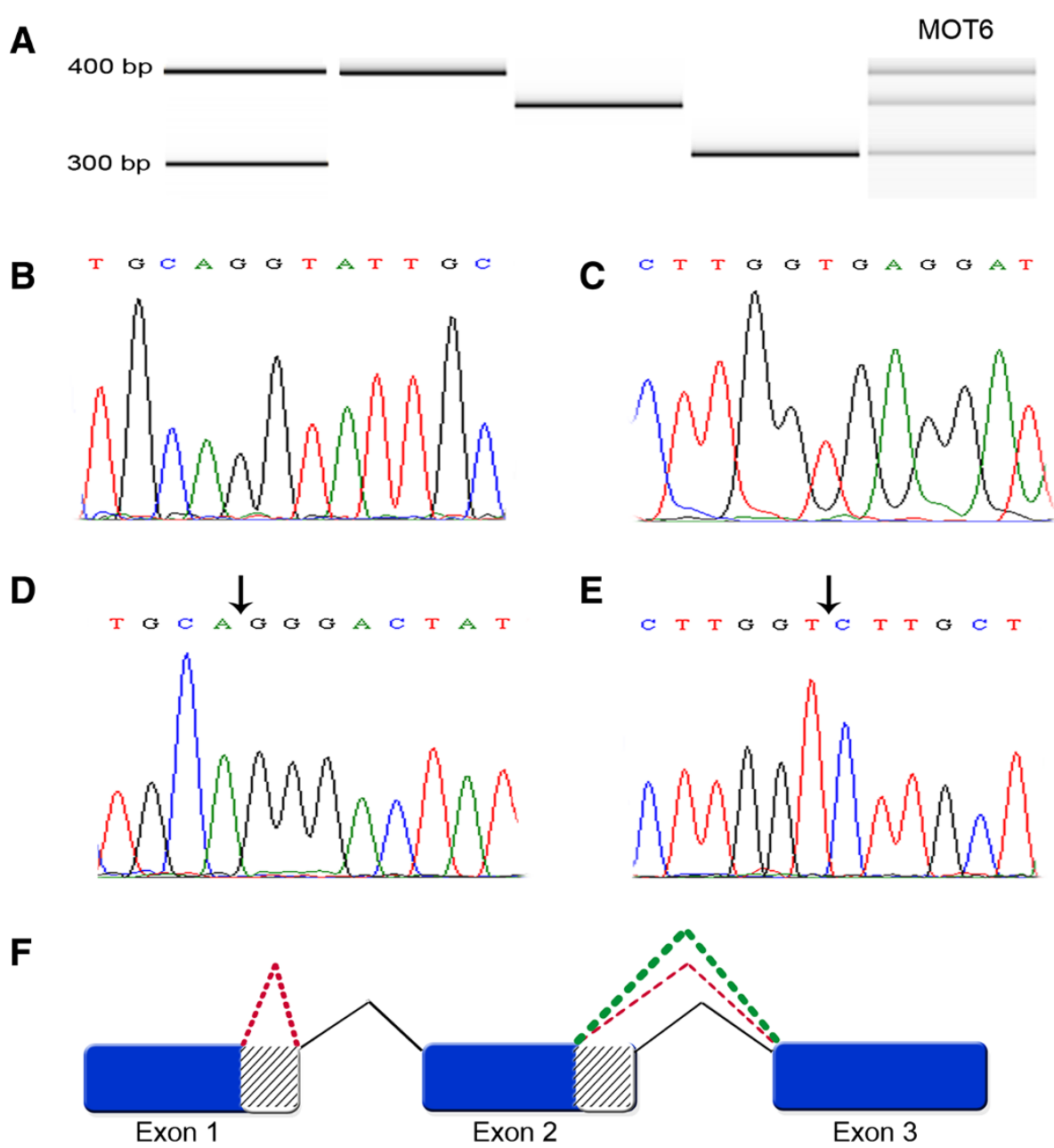

Figure 5 Cloning and sequencing of novel ZNF695 transcripts associated to ovarian cancer. A) PCR product of ZNF695 showing the three different RNA transcript sizes of ZNF695 expressed in malignant ovary tissue sample 6 (MOT6, right) and the individual amplicons used for cloning, with the size (in base pairs) shown on the left. Ladder (line 1), ZNF695 splice variants 1 and/or 2 (lane 2, 400 bp), ZNF695 splice variant 4 (lane 3, 360 bp), ZNF695 splice variant 5 (lane 4, 310 bp), and ZNF695 amplicons in MOT6 (line 5). B) Sanger sequence plot of ZNF695 splice variant 1 and 2 at the boundary of exons 1 and 2. C) Sanger sequence plot of ZNF695 splice variant 1 and 2 at the boundary of exons 2 and 3. D) Sanger sequence plot of ZNF695 splice 5. This transcript has alternative splicing in exon 1-2, with the arrow illustrating the alternative splicing site at the exon 1-2 boundary. E) Sanger sequence plot of ZNF695 splice variant 4. This sequence has alternative site splicing in exon 2-3 (arrows), this sequence are contain in ZNF695 variant 5 too. F) AS model of ZNF695 gene. Black lines represent primary splicing of exons 1-2-3 of ZNF695 gene containing the full coding sequence that corresponds to the largest amplicon. Green dotted line shows exons 2-3 AS, which corresponds to the intermediate amplicon. Red dotted line shows exon 1 AS together with exons 2-3 AS, which are contained in the smaller amplicon.

basis of malignant transformation, tumor behavior [23,41], and even for the identification of potential therapeutic targets [42].

We found that OC tissue has indeed a signature of alternatively spliced genes. Although we do not know yet whether these changes are indeed related only to OC or they are general markers of cancer. Of the $>270$ differentially spliced genes found in $\mathrm{OC}$, nine were highly significant, but we decided to focus on the most significantly expressed gene with differential AS, the zinc finger protein ZNF695.

The zinc finger protein family (ZNF) spans over 700 members with many functional roles within the cell, including regulation of gene expression, which is achieved by different means. For instance, $\mathrm{ZNF}$ transcription factors bind to DNA by means of $\mathrm{C}_{2} \mathrm{H}_{2}$ zinc finger domains, constituting a subfamily of ZNF [43]. Although some ZNF members act only as repressors, others solely as act as activators, most of them can apparently be either repressors or activators depending on the particular status of the cell. Moreover, some ZNF play roles in signal transduction and many other cellular functions. ZNF695, which we found here to be differentially expressed and spliced in OC belongs to the $\mathrm{C}_{2} \mathrm{H}_{2}$ subfamily of ZNF and also contains Krüpple-associated box (KRAB) domains, which characteristically identify gene repressors [44-46]. 
Because these genes, including ZNF695 contain two or more functional domains, changes affecting only one domain can have dramatic consequences [45,47]. On one hand, repressors could lose their regulatory function or even turn in the opposite direction and become activators [47]. KRAB domains in ZNF proteins serve to bind corepressors, which in turn mediate transcription repression [44]. Of the ZNF695 splice variants we found here to be preferentially associated to $\mathrm{OC}$, isoforms 2 and 3 have incomplete KRAB domains that are essential for interactions with co-repressors which suggests that such AS pattern could be related to carcinogenesis. The third variant lacked the initial translation codon; hence, it is unlikely to yield a translational product.

Unfortunately, as yet, almost nothing is known about ZNF695 in humans or in other species, with the closest homologues having up to $64 \%$ identity. Therefore, at present it is not possible to predict how ZNF695 could play a role in OC development, if at all. One could envision that because the alternative forms found in $\mathrm{OC}$ have incomplete KRAB domains, this potential repressor could function as an activator and turn on cell proliferation and, hence, malignant transformation. The other possibility would be to function as dominant negative variants, but this seems unlikely because normal ovarian tissue does not express ZNF695 in any of its isoforms ad OC cells express only the alternative splice variants. Therefore, we consider ZNF695 splice variants $1 / 2,4$ and 5 as potential oncogenes playing a role in the pathogenesis of OC.

\section{Additional files}

\section{Additional file 1: Graphic showing the proportions of four} microarray quality controls. The $Y$-axis depicts the expression level of controls (Log2) and the $X$ - axis contains the 14 samples used for these studies. A) Microarray hybridization controls where the purple line corresponds to the control (CreX), green (BioDn), blue (BioC) red (BioB). B) Microaray labelling controls: pink line (dab), brown (thr), blue (phe), and orange controls (lys).

Additional file 2: Table of genes expressed in ovarian samples. Additional file 3: Table of significantly over-expressed genes with potential alternative splicing.

Additional file 4: Alignment of ZNF695 splice variants. BLAST analysis of sequences obtained from the clones, alternatively spliced ZNF695 variants found predominantly in malignant ovarian tissue and cell lines. Transcript variant 1/2, the sequence corresponds to the heavier band. ZNF695 Transcript variant 4, the sequence corresponds to the medium sized band, and ZNF695 splice variant 5, this sequence corresponds to the lighter band.

Additional file 5: Alignment of predicted ZNF695 peptides. In silico translation of ZNF695 amplicons (ZNF695 transcript variant_1/2, ZNF695 transcript variant 4, ZNF695 transcript variant 5) revealing sequence identity of unnamed protein product (BAG54313.1) with ZNF695 transcript variants 4 and 5 . ZNF695 transcript variant $1 / 2$ is identical to full length ZNF695 (transcript variant 1 or 2).

\section{Abbreviations}

OC: Ovarian cancer; AS: Alternative splicing; HOT: Health ovarian tissue; BOT: Borderline ovarian tumor; MOT: Malignant ovarian tumor; OCL: Ovarian cell lines; PCA: Principal component analysis; QC: Quality control; FDR: False discovery ratio; FLP: Full-length product; KRAB: Krüpple-associated box.

\section{Competing interests}

The authors declare that they have no competing interests.

\section{Authors' contributions}

SJ-M participated in the study design, carried out experiments, performed statistical analysis and helped to draft the manuscript, W-R and RL-R performed experimental procedures, OAP-G, and MS participated in microarray analysis, VEMO-F, AZ-D participated in sequencing and cloning experiments, ML-O, RB, provided clinical sample material, performed the pathological analysis and tumor classification. JM participated in the design of the study, coordination and helped to draft the manuscript, GV-O conceived the study and participated in its design and helped to draft the manuscript. All authors read and approved the final manuscript.

\section{Acknowledgements}

This work was supported in part by a Basic Science grant 61742 form SEP-CONACYT México. Sergio Juárez-Méndez was financially supported by a scholarship provided by the National Council of Science and Technology (CONACYT) and IMSS. This work constitutes a partial fulfillment of the graduate program in PhD Biological Science of National Autonomous University of México (UNAM) of Sergio Juárez-Méndez. We finally thank Dr. Carlos Pérez-Plasencia, Faculty of Superior Studies, Ixtacala, UNAM for critical review of the manuscript and Dr. Laura Díaz-Cueto, Research Unit on Reproductive Medicine, Instituto Mexicano del Seguro Social (IMSS) for kindly providing cell lines.

\section{Author details}

'Experimental Oncology Laboratory, Research Department, National Institute of Pediatrics, Mexico, D. F, Mexico. ${ }^{2}$ Mexican Faculty of Medicine, Universidad La Salle, Mexico City, Mexico. ${ }^{3}$ Genomic Medicine and Environmental Toxicology Department, Biomedical Research Institute, UNAM, Mexico, D. F, Mexico. ${ }^{4}$ Unit of Biochemistry, National Institute of Medical Sciences and Nutrition "Salvador Zubirán", Mexico City, Mexico. ${ }^{5}$ Genomic Oncology Laboratory, Medical Research Unit in Oncologic Diseases, Oncology Hospital, National Medical Center Siglo XXI, IMSS, Mexico City, Mexico. ${ }^{6}$ Faculty of Medicine, UNAM, General Hospital of México O. D, Mexico, D. F, Mexico. ${ }^{7}$ Department of Oncology, General Hospital of México O. D, Mexico, D. F, Mexico. ${ }^{8}$ Mammalian Genetics Section, NIDDK, National Institutes of Health, 10/9 N104, 10 Center Drive, Bethesda, MD 20892, USA.

Received: 28 May 2013 Accepted: 24 August 2013

Published: 5 September 2013

\section{References}

1. Bast RC Jr, Hennessy B, Mills GB: The biology of ovarian cancer: new opportunities for translation. Nat Rev Cancer 2009, 9:415-428.

2. Scully RE: Classification of human ovarian tumors. Env Health Perspect 1987, 73:15-25.

3. Lancaster JM, Dressman HK, Clarke JP, Sayer RA, Martino MA, Cragun JM, Henriott AH, Gray J, Sutphen R, Elahi A, et al: Identification of genes associated with ovarian cancer metastasis using microarray expression analysis. Int J Gynecol Cancer 2006, 16:1733-1745.

4. Maxwell GL, Chandramouli GV, Dainty L, Litzi TJ, Berchuck A, Barrett JC, Risinger Jl: Microarray analysis of endometrial carcinomas and mixed mullerian tumors reveals distinct gene expression profiles associated with different histologic types of uterine cancer. Clin Cancer Res 2005, 11:4056-4066.

5. Tinker AV, Boussioutas A, Bowtell DD: The challenges of gene expression microarrays for the study of human cancer. Cancer Cell 2006, 9:333-339.

6. Zorn KK, Bonome T, Gangi L, Chandramouli GV, Awtrey CS, Gardner GJ, Barrett JC, Boyd J, Birrer MJ: Gene expression profiles of serous, endometrioid, and clear cell subtypes of ovarian and endometrial cancer. Clin Cancer Res 2005, 11:6422-6430.

7. Keita M, Bachvarova M, Morin C, Plante M, Gregoire J, Renaud MC, Sebastianelli A, Trinh XB, Bachvarov D: The RUNX1 transcription factor is expressed in serous epithelial ovarian carcinoma and contributes to cell proliferation, migration and invasion. Cell Cycle 2013, 12:972-986. 
8. Treeck O, Schuler S, Haring J, Skrzypczak M, Lattrich C, Ortmann O: icb-1 Gene counteracts growth of ovarian cancer cell lines. Cancer Lett 2013, 335:441-446.

9. Lander ES, Linton LM, Birren B, Nusbaum C, Zody MC, Baldwin J, Devon K, Dewar K, Doyle M, FitzHugh W, et al: Initial sequencing and analysis of the human genome. Nature 2001, 409:860-921

10. Modrek B, Resch A, Grasso C, Lee C: Genome-wide detection of alternative splicing in expressed sequences of human genes. Nucleic Acids Res 2001 29:2850-2859.

11. Graveley BR: Alternative splicing: increasing diversity in the proteomic world. Trends Genet 2001, 17:100-107.

12. Carninci P: Constructing the landscape of the mammalian transcriptome. J Exp Biol 2007, 210:1497-1506.

13. Schmucker D, Clemens JC, Shu H, Worby CA, Xiao J, Muda M, Dixon JE, Zipursky SL: Drosophila Dscam is an axon guidance receptor exhibiting extraordinary molecular diversity. Cell 2000, 101:671-684.

14. Venables JP: Unbalanced alternative splicing and its significance in cancer. Bioessays 2006, 28:378-386.

15. Hayes GM, Carrigan PE, Miller LJ: Serine-arginine protein kinase 1 overexpression is associated with tumorigenic imbalance in mitogen-activated protein kinase pathways in breast, colonic, and pancreatic carcinomas. Cancer Res 2007, 67:2072-2080.

16. Akgul C, Moulding DA, Edwards SW: Alternative splicing of Bcl-2-related genes: functional consequences and potential therapeutic applications. Cell Mol Life Sci 2004, 61:2189-2199.

17. Chen LL, Sabripour M, Wu EF, Prieto VG, Fuller GN, Frazier ML: A mutation-created novel intra-exonic pre-mRNA splice site causes constitutive activation of KIT in human gastrointestinal stromal tumors. Oncogene 2005, 24:4271-4280.

18. Wang XQ, Luk JM, Leung PP, Wong BW, Stanbridge EJ, Fan ST: Alternative mRNA splicing of liver intestine-cadherin in hepatocellular carcinoma. Clin Cancer Res 2005, 11:483-489.

19. Narla G, Difeo A, Reeves HL, Schaid DJ, Hirshfeld J, Hod E, Katz A, Isaacs WB, Hebbring S, Komiya A, et al: A germline DNA polymorphism enhances alternative splicing of the KLF6 tumor suppressor gene and is associated with increased prostate cancer risk. Cancer Res 2005, 65:1213-1222.

20. Thorsen K, Sorensen KD, Brems-Eskildsen AS, Modin C, Gaustadnes M, Hein AM, Kruhoffer M, Laurberg S, Borre M, Wang K, et al: Alternative splicing in colon, bladder, and prostate cancer identified by exon array analysis. Mol Cell Proteomics 2008, 7:1214-1224.

21. Adamia S, Reiman T, Crainie M, Mant MJ, Belch AR, Pilarski LM: Intronic splicing of hyaluronan synthase 1 (HAS1): a biologically relevant indicator of poor outcome in multiple myeloma. Blood 2005, 105:4836-4844.

22. Mazoyer S, Puget N, Perrin-Vidoz L, Lynch HT, Serova-Sinilnikova OM Lenoir GM: A BRCA1 nonsense mutation causes exon skipping. Am J Hum Genet 1998, 62:713-715.

23. Singh A, Karnoub AE, Palmby TR, Lengyel E, Sondek J, Der CJ: Rac1b, a tumor associated, constitutively active Rac1 splice variant, promotes cellular transformation. Oncogene 2004, 23:9369-9380

24. Zhou YQ, He C, Chen YQ, Wang D, Wang MH: Altered expression of the RON receptor tyrosine kinase in primary human colorectal adenocarcinomas: generation of different splicing RON variants and their oncogenic potential. Oncogene 2003, 22:186-197.

25. Bauer TW, Fan F, Liu W, Johnson M, Parikh NU, Parry GC, Callahan J, Mazar AP, Gallick GE, Ellis LM: Insulinlike growth factor-l-mediated migration and invasion of human colon carcinoma cells requires activation of c-Met and urokinase plasminogen activator receptor Ann Surg 2005, 241:748-756. discussion 756-748.

26. Brembeck FH, Rosario M, Birchmeier W: Balancing cell adhesion and Wnt signaling, the key role of beta-catenin. Curr Opin Genet Dev 2006, 16:51-59.

27. Cheng C, Sharp PA: Regulation of CD44 alternative splicing by $\mathrm{SRm} 160$ and its potential role in tumor cell invasion. Mol Cell Biol 2006, 26:362-370.

28. Wong MP, Cheung N, Yuen ST, Leung SY, Chung LP: Vascular endothelial growth factor is up-regulated in the early pre-malignant stage of colorectal tumour progression. Int J Cancer 1999, 81:845-850.

29. Hofstetter G, Berger A, Fiegl H, Slade N, Zoric A, Holzer B, Schuster E, Mobus VJ, Reimer D, Daxenbichler G, et al: Alternative splicing of p53 and p73: the novel p53 splice variant p53delta is an independent prognostic marker in ovarian cancer. Oncogene 2010, 29:1997-2004.

30. Hamilton TC, Young RC, McKoy WM, Grotzinger KR, Green JA, Chu EW, Whang-Peng J, Rogan AM, Green WR, Ozols RF: Characterization of a human ovarian carcinoma cell line (NIH: OVCAR-3) with androgen and estrogen receptors. Cancer Res 1983, 43:5379-5389.

31. Fogh J, Wright WC, Loveless JD: Absence of HeLa cell contamination in 169 cell lines derived from human tumors. J Natl Cancer Inst 1977, 58:209-214

32. Provencher DM, Lounis $H$, Champoux L, Tetrault M, Manderson EN, Wang JC, Eydoux P, Savoie R, Tonin PN, Mes-Masson AM: Characterization of four novel epithelial ovarian cancer cell lines. In vitro Cell Dev Biol Anim 2000, 36:357-361.

33. Hoskins WJ: Prospective on ovarian cancer: why prevent? J Cell Biochem Suppl 1995, 23:189-199.

34. Nguyen HN, Averette HE, Hoskins W, Sevin BU, Penalver M, Steren A National survey of ovarian carcinoma. VI. Critical assessment of current International Federation of Gynecology and Obstetrics staging system. Cancer 1993, 72:3007-3011.

35. Venables JP, Klinck R, Koh C, Gervais-Bird J, Bramard A, Inkel L, Durand M, Couture S, Froehlich U, Lapointe $\mathrm{E}$, et al: Cancer-associated regulation of alternative splicing. Nat Struct Mol Biol 2009, 16:670-676.

36. Zardi L, Carnemolla B, Siri A, Petersen TE, Paolella G, Sebastio G, Baralle FE: Transformed human cells produce a new fibronectin isoform by preferential alternative splicing of a previously unobserved exon. EMBO J 1987, 6:2337-2342.

37. Missler M, Sudhof TC: Neurexins: three genes and 1001 products. Trends Genet 1998, 14:20-26.

38. Joseph R, Dou D, Tsang W: Neuronatin mRNA: alternatively spliced forms of a novel brain-specific mammalian developmental gene. Brain Res 1995, 690:92-98.

39. Chen $C D$, Kobayashi $\mathrm{R}$, Helfman DM: Binding of hnRNP $\mathrm{H}$ to an exonic splicing silencer is involved in the regulation of alternative splicing of the rat beta-tropomyosin gene. Genes Dev 1999, 13:593-606.

40. Merdzhanova G, Gout S, Keramidas M, Edmond V, Coll JL, Brambilla C, Brambilla E, Gazzeri S, Eymin B: The transcription factor E2F1 and the SR protein SC35 control the ratio of pro-angiogenic versus antiangiogenic isoforms of vascular endothelial growth factor-A to inhibit neovascularization in vivo. Oncogene 2010, 29:5392-5403.

41. Guo M, Liu W, Serra S, Asa SL, Ezzat S: FGFR2 isoforms support epithelial-stromal interactions in thyroid cancer progression. Cancer Res 2012, 72:2017-2027.

42. Dery KJ, Gusti V, Gaur S, Shively JE, Yen Y, Gaur RK: Alternative splicing as a therapeutic target for human diseases. Methods Mol Biol 2009, 555:127-144.

43. Krishna SS, Majumdar I, Grishin NV: Structural classification of zinc fingers: survey and summary. Nucleic Acids Res 2003, 31:532-550.

44. Friedman JR, Fredericks WJ, Jensen DE, Speicher DW, Huang XP, Neilson EG, Rauscher FJ 3rd: KAP-1, a novel corepressor for the highly conserved KRAB repression domain. Genes Dev 1996, 10:2067-2078.

45. Vissing $H$, Meyer WK, Aagaard L, Tommerup N, Thiesen HJ: Repression of transcriptional activity by heterologous KRAB domains present in zinc finger proteins. FEBS Lett 1995, 369:153-157.

46. Pengue G, Calabro V, Bartoli PC, Pagliuca A, Lania L: Repression of transcriptional activity at a distance by the evolutionarily conserved KRAB domain present in a subfamily of zinc finger proteins. Nucleic Acids Res 1994, 22:2908-2914.

47. Agata Y, Matsuda E, Shimizu A: Two novel Kruppel-associated box-containing zinc-finger proteins, KRAZ1 and KRAZ2, repress transcription through functional interaction with the corepressor KAP-1 (TIF1beta/KRIP-1) J Biol Chem 1999, 274:16412-16422.

doi:10.1186/1757-2215-6-61

Cite this article as: Juárez-Méndez et al:: Splice variants of zinc finger protein 695 mRNA associated to ovarian cancer. Journal of Ovarian Research 2013 6:61. 